\title{
Usefulness of the Comprehensive Geriatric Assessment in Older Patients with Upper Gastrointestinal Bleeding: A Two-Year Follow-Up Study
}

\author{
Alberto Pilotto $^{a}$ Luigi Ferrucci $^{\mathrm{e}}$ Carlo Scarcelli $^{\mathrm{a}}$ Valeria Niro $^{\mathrm{a}}$ \\ Francesco Di Mario $^{c}$ Davide Seripa ${ }^{a}$ Angelo Andriullib ${ }^{b}$ Gioacchino Leandro $^{d}$ \\ Marilisa Franceschi ${ }^{a}$ c \\ ${ }^{a}$ Geriatric Unit and Gerontology \& Geriatrics Research Laboratories, and b Gastroenterology Unit, IRCCS 'Casa \\ Sollievo della Sofferenza', San Giovanni Rotondo, 'Gastroenterology Department, University of Parma, Parma,

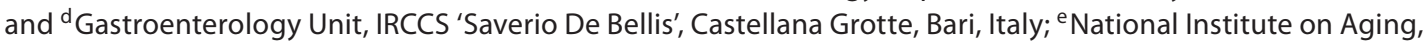 \\ Longitudinal Studies Section, Harbor Hospital Center, Baltimore, Md., USA
}

\section{Key Words \\ Comprehensive geriatric assessment $\cdot$ Multidimensional prognostic index $\cdot$ Upper gastrointestinal bleeding, diagnosis}

\begin{abstract}
Background: The potential usefulness of standardized comprehensive geriatric assessment (CGA) in evaluating treatment and follow-up of older patients with upper gastrointestinal bleeding is unknown. Aim: To evaluate the usefulness of the CGA as a 2-year mortality multidimensional prognostic index (MPI) in older patients hospitalized for upper gastrointestinal bleeding. Materials and Methods: $\mathrm{Pa}$ tients aged $\geq 65$ years consecutively hospitalized for acute upper gastrointestinal bleeding were included. Diagnosis of bleeding was based on clinical and endoscopic features. All patients underwent a CGA that included six standardized scales, i.e., Activities of Daily Living (ADL), Instrumental Activities of Daily Living (IADL), Short Portable Mental Status Questionnaire (SPMSQ), Mini Nutritional Assessment (MNA), Exton-Smith Score (ESS) and Comorbity Index Rating Scale (CIRS), as well as information on medication history and cohabitation, for a total of 63 items. A MPI was calculated from
\end{abstract}

the integrated total scores and expressed as MPI $1=$ low risk, $\mathrm{MPI} 2$ = moderate risk, and MPI 3 = severe risk. The predictive value of the MPI for mortality over a 24-month follow-up was calculated. Results: 36 elderly patients (M 16/F 20, mean age $82.8 \pm 7.9$ years, range 70-101 years) were included in the study. A significant difference in mean age was observed between males and females (M $80.1 \pm 4.8$ vs. F $84.9 \pm 9.3$ years; $p<0.05$ ). The causes of upper gastrointestinal bleeding were duodenal ulcer in $38.8 \%$, gastric ulcer in $22.2 \%$, and erosive gastritis in $16.6 \%$ of the patients, while $16.6 \%$ had gastrointestinal bleeding from unknown origin. The overall 2-year mortality rate was $30.5 \% .18$ patients $(50 \%)$ were classified as having a low-risk MPI (mean value $0.18 \pm 0.09$ ), 12 (33.3\%) as having a moderate-risk MPI (mean value $0.48 \pm$ 0.08 ) and $6(16.6 \%)$ as having a severe-risk MPI (mean value $0.83 \pm 0.06)$. Higher MPI grades were significantly associated with higher mortality (grade $1=12.5 \%$, grade $2=41.6 \%$, grade $3=83.3 \% ; p=0.001$ ). Adjusting for age and sex, the prognostic efficacy of MPI for mortality was confirmed and highly significant (odds ratio $10.47,95 \% \mathrm{Cl} 2.04-53.6$ ). Conclusion: CGA is a useful tool for calculating a MPI that significantly predicts the risk of 2-year mortality in older patients with upper gastrointestinal bleeding.

Copyright $\odot 2007$ S. Karger AG, Basel

\section{KARGER}

Fax +4161306 1234

E-Mail karger@karger.ch

www.karger.com (c) 2007 S. Karger AG, Basel

0257-2753/07/0252-0124\$23.50/0

Accessible online at:

www.karger.com/ddi
Alberto Pilotto

Unità Operativa di Geriatria, Ospedale 'Casa Sollievo della Sofferenza'

Istituto di Ricovero e Cura a Carattere Scientifico

IT-71013 San Giovanni Rotondo (Italy)

Tel./Fax +39088241 0271, E-Mail alberto.pilotto@libero.it 


\section{Introduction}

Comprehensive geriatric assessment (CGA) is a multidimensional, usually interdisciplinary, diagnostic process intended to determine an elderly person's medical, psychosocial, and functional capacity and problems with the objective of developing an overall plan for treatment and long-term follow-up. It is particularly useful in dealing with frail elderly, since such patients are likely to have multiple and interacting problems that interfere with daily functioning and complicate treatment, all of which can be better understood and addressed through the comprehensive assessment process. The process of geriatric assessment can range in intensity from a limited assessment by primary care physicians or community health workers focused on identifying an older person's functional problems and disabilities (screening assessment), to more complete evaluations of these problems usually coupled with therapeutic plans by a multidisciplinary team with geriatric training and experience (comprehensive geriatric assessment).

Indeed, an older patient presenting with a gastrointestinal problem typically has a multitude of other disease conditions and age-related disorders that complicate the diagnostic and therapeutic options and make the comprehensive assessment approach particularly valuable [1] A large number of studies support the effectiveness of geriatric assessment programs in a variety of settings [2, $3]$. However, no data on the use of the CGA in older patients with upper gastrointestinal bleeding have been reported.

The aim of this study was to evaluate the usefulness of the CGA as a 2-year mortality multidimensional prognostic index (MPI) in older patients hospitalized for upper gastrointestinal bleeding.

\section{Materials and Methods}

\section{Subjects}

The study was conducted according to the Declaration of Helsinki and the guidelines for Good Clinical Practice and was approved by our Institution Ethics Committee. Written informed consent was obtained from the patients or from relatives of critically ill or demented patients prior to participation in the study.

All patients aged $\geq 65$ years consecutively admitted from January 1 to December 31, 2004, to the Geriatric Unit of the Casa Sollievo della Sofferenza Hospital (IRCCS, San Giovanni Rotondo, Italy) for acute upper gastrointestinal bleeding were screened for study inclusion. Inclusion criteria were: (1) age $\geq 65$ years; (2) diagnosis of upper gastrointestinal bleeding; (3) complete
CGA during hospitalization, and (4) availability of mortality/survival information at the date of study completion.

At baseline, demographic and clinical parameters were defined by structured interview, clinical evaluation and review of records from the patients' general practitioners: date of birth, gender, clinical history, current pathologies and medication history. Vital status up to December 31, 2005, was assessed by directly contacting the participants or consulting the Registry Offices of the cities where the patients were residents at the time of hospital admission. Dates of death were identified from death certificates.

\section{Diagnosis of Upper Gastrointestinal Bleeding}

Endoscopic diagnoses for esophageal, gastric and duodenal lesions were based on the criteria of Cotton and Williams [4]. Upper gastrointestinal tract bleeding was diagnosed on the basis of clinical signs (hematemesis, melena or anemia with a loss of $>3 \mathrm{~g}$ of hemoglobin) and endoscopic stigmata of a recent hemorrhage defined according to Laine and Peterson [5] as a flat pigmented spot (red, purple, brown or black), an adherent clot, a visible vessel or active bleeding. Patients with bleeding from esophageal varices and/or coagulation diseases were excluded.

\section{Comprehensive Geriatric Assessment}

CGA was carried out including assessment instruments that are widely used in geriatric practice. Functional status was evaluated by the Activities of Daily Living (ADL) index [6], which defines the level of dependence/independence of 6 daily personal care activities including bathing, toileting, feeding, dressing, urine and bowel continence and transferring (in and out of bed or chair) and by the Instrumental Activities of Daily Living (IADL) scale [7], which assesses independence in 8 activities that are more cognitively and physically demanding than ADL, including managing finances, taking medications, using the telephone, shopping, using transportation, preparing meals, doing housework and washing.

Cognitive status was assessed by the Short Portable Mental Status Questionnaire (SPMSQ), a 10-item questionnaire that assesses orientation, memory, attention, calculation and language [8].

Comorbidity was examined using the Cumulative Illness Rating Scale (CIRS) [9]. The CIRS uses 5-point ordinal scales (score $1-5)$ to estimate the severity of pathology in each of 13 systems, including cardiac, vascular, respiratory, eye-ear-nose-throat, upper and lower gastroenterological diseases, hepatic, renal, genitourinary, musculoskeletal, skin disorders, nervous system, endocrine-metabolic and psychiatric behavioral problems. Based on the ratings, the two following scores are derived: (a) the Comorbidity Index (CIRS-CI) score, which reflects the number of concomitant diseases, and is derived from the total number of categories in which moderate or severe levels (grades from 3 to 5) of disease are quoted (range from 0 to 13), and (b) the Severity Index (CIRS-SI), which reflects the overall severity of diseases, and the average rating of 13 disease categories, excluding psychiatric behavioral problems (range from 1 to 5).

Nutritional status was explored with the Mini Nutritional Assessment (MNA) [10], which includes information on (a) anthropometric measures (body mass index, BMI: body weight $/$ height $^{2}$, mid-arm circumference in $\mathrm{cm}, \mathrm{MAC}$, calf circumference in $\mathrm{cm}$, CC, and weight loss); (b) lifestyle, medication and mobility; 
Table 1. Patient characteristics divided according to gender

\begin{tabular}{|c|c|c|c|c|}
\hline & $\begin{array}{l}\text { All } \\
(n=36)\end{array}$ & $\begin{array}{l}\text { Males } \\
(\mathrm{n}=16)\end{array}$ & $\begin{array}{l}\text { Females } \\
(n=20)\end{array}$ & $\mathrm{p}$ \\
\hline Age (mean $\pm S D)$, years & $82.8 \pm 7.9$ & $80.1 \pm 4.8$ & $84.9 \pm 9.3$ & 0.03 \\
\hline Age, range & $70-101$ & $70-90$ & $70-101$ & \\
\hline Education level (mean $\pm S D)$, years & $4.4 \pm 3.9$ & $5.2 \pm 5.3$ & $3.6 \pm 2.0$ & 0.426 \\
\hline $\mathrm{ADL}$ score $($ mean $\pm \mathrm{SD})$ & $4.1 \pm 2.4$ & $4.3 \pm 2.5$ & $3.9 \pm 2.3$ & 0.481 \\
\hline IADL score $($ mean \pm SD $)$ & $3.9 \pm 3.2$ & $4.4 \pm 3.5$ & $3.6 \pm 3.1$ & 0.386 \\
\hline SPMSQ score (mean \pm SD) & $2.7 \pm 3.4$ & $2.9 \pm 3.3$ & $2.4 \pm 2.9$ & 0.808 \\
\hline Exton-Smith score (mean $\pm \mathrm{SD})$ & $15.3 \pm 4.5$ & $15.4 \pm 5.0$ & $15.1 \pm 4.1$ & 0.445 \\
\hline CIRS comorbidity score (mean $\pm \mathrm{SD}$ ) & $3.1 \pm 2.1$ & $3.3 \pm 2.6$ & $2.9 \pm 1.5$ & 0.854 \\
\hline MNA score $($ mean $\pm \mathrm{SD})$ & $19.2 \pm 7.0$ & $20.7 \pm 7.9$ & $18.1 \pm 6.3$ & 0.180 \\
\hline Drugs, $\mathrm{n}($ mean $\pm \mathrm{SD})$ & $3.4 \pm 1.9$ & $3.5 \pm 1.4$ & $3.3 \pm 2.2$ & 0.539 \\
\hline Low-dose aspirin/NSAID, n (\%) & $11(30.6)$ & $6(37.5)$ & $5(25.0)$ & 0.656 \\
\hline Warfarin, $\mathrm{n}(\%)$ & $3(8.3)$ & $2(12.5)$ & $1(5.0)$ & 0.840 \\
\hline PPI, n (\%) & $6(16.7)$ & $2(12.5)$ & $4(20)$ & 0.881 \\
\hline $\mathrm{MPI}($ mean $\pm \mathrm{SD})$ score & $0.39 \pm 0.26$ & $0.36 \pm 0.27$ & $0.40 \pm 0.25$ & 0.560 \\
\hline Mortality, n (\%) & $11(30.6)$ & $3(18.8)$ & $8(40.0)$ & 0.156 \\
\hline
\end{tabular}

(c) number of meals, food and fluid intake, autonomy of feeding, and (4) self-perception of health and nutrition.

The Exton-Smith Scale (ESS) was used to evaluate the risk of developing pressure sores. This 5 -item questionnaire determines physical condition, mental condition, activity, mobility and incontinence. For each item, a score from 1 to 4 is assigned [11].

Medication use was defined according to the Anatomical Therapeutics Chemical Classification code system (ATC classification) [12] and the number of drugs used by patients at admission was recorded. Patients were defined as drug users if they took a medication of any of the above-described classes at the moment of admission.

Social aspects include household composition, home services, and institutionalization.

\section{Multidimensional Prognostic Index}

The MPI was calculated by aggregating data from specific questionnaires (ADL, IADL, SPMSQ, CIRS-CI, MNA, EES) as well as additional data on medication and cohabitation status, for a total of 63 items. All scales were arbitrarily reduced to three values, i.e., $0=$ no problems, $0.5=$ minor problems, and $1=$ major problems based in part on data from the literature and a general discussion among the members of the team that occurred prior to any data analysis. The sum of the calculated scores from the above-reported eight domains was divided by 8 to obtain a final MPI score from 0 to 1 . Three levels of risk were arbitrarily identified: MPI 1 = low risk, range 0.0-0.33; MPI $2=$ moderate risk, range $0.34-0.66$, and MPI 3 = severe risk, range $0.67-1.0$ as previously reported [13].

\section{Statistical Analysis}

All analyses were performed using the SPSS Version 13 software for Windows (SPSS Inc., Chicago, Ill., USA) [14]. Continuous variables are shown as mean $\pm \mathrm{SD}$ and comparisons between men and women were defined using the Mann-Whitney U-test. The Kruskal-Wallis analysis of variance was used to compare mortality rate across MPI groups.
The relationship between MPI score group and time-to-death was analyzed by an age- and sex-adjusted Cox proportional hazard regression model. Time to death was calculated as the time between admission and time of death or end of follow-up whichever came first. The proportionally of the hazard assumption was graphically checked by plotting $\log [-\log$ (survival function) $]$. A p value of $<0.05$ was considered statistically significant.

\section{Results}

During the 1-year inclusion period, 52 patients with a diagnosis of upper gastrointestinal bleeding were consecutively admitted to our Unit. Eight patients were excluded because the CGA was not completed, 4 patients were excluded because information on their vital status at study conclusion was not available, and 4 patients were excluded because they refused to participate in the study. Thus, the final study population included 36 elderly patients: 16 men and 20 women with a mean age of $82.8 \pm$ 7.9 years, and a range of 70-101 years.

Table 1 reports the characteristics of patients included in the study. A significant difference in mean age was observed between males and females (M $80.1 \pm 4.8$ vs. F $84.9 \pm 9.3$ years; $\mathrm{p}=0.03)$. No differences were found between males and females for all other parameters (ADL, IADL, SPMSQ, MNA, ESS, CIRS-CI, drug use and cohabitation status). Table 2 shows the endoscopic diagnoses of patients: $38.8 \%$ had duodenal ulcer, $22.2 \%$ had gastric ulcer, $16.6 \%$ had erosive gastritis, and $16.6 \%$ had gastrointestinal bleeding from unknown origin. The overall 2 -year mortality rate was $30.6 \%$. 
Fig. 1. Survival curves of elderly patients

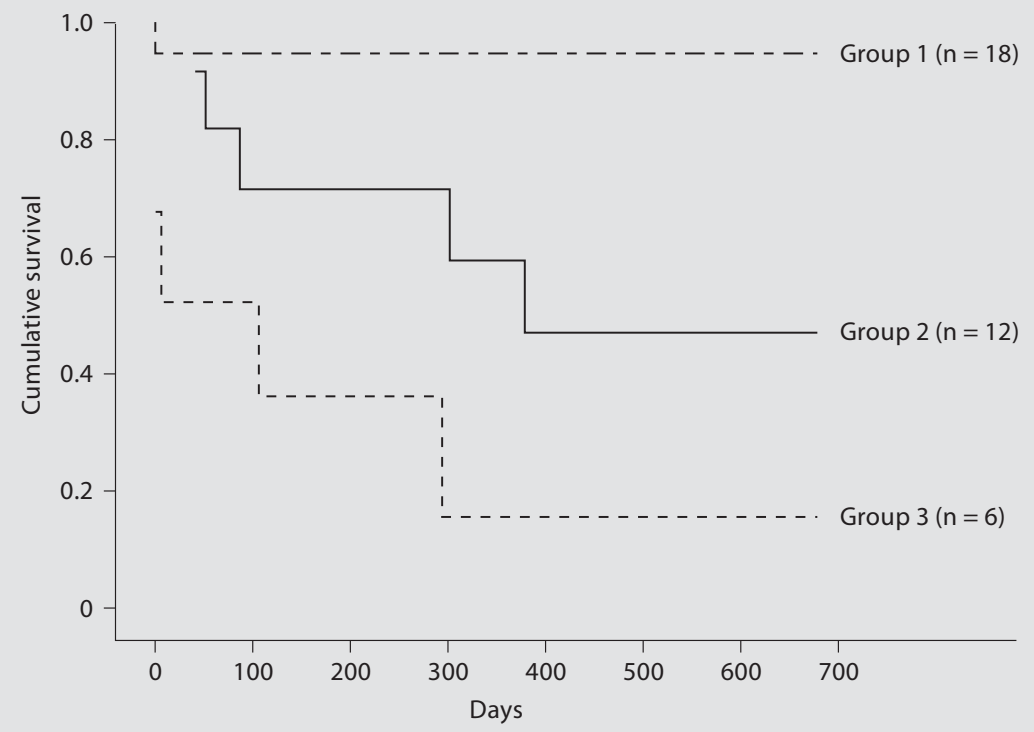
$(\mathrm{n}=36)$ with three grades of MPI for 2-year mortality as derived from a CGA.

Table 2. Endoscopic diagnoses of bleeding patients $(n=36)$ included in the study

\begin{tabular}{lc}
\hline Diagnosis & Patients (\%) \\
\hline Esophageal ulcer & $1(2.8)$ \\
Erosive gastritis & $6(16.6)$ \\
Gastric ulcer & $8(22.2)$ \\
Duodenal ulcer & $14(38.8)$ \\
Duodenal angiodysplasia & $1(2.8)$ \\
Unknown origin GI bleeding & $6(16.6)$ \\
\hline
\end{tabular}

The mean MPI value in the total study population was $0.39 \pm 0.26$. Overall, 18 patients, i.e., $50 \%$ of the total study population, were included in the MPI grade 1 group (low risk, MPI mean value $0.18 \pm 0.09$ ); 12 patients, i.e., $33.3 \%$ of the study population, in the MPI grade 2 (moderate risk, MPI mean value $0.48 \pm 0.08$ ) and 6 patients, i.e., $16.6 \%$ of the total population, in the MPI grade 3 (severe risk, MPI mean value $0.83 \pm 0.06$ ). Higher MPI grades were significantly associated with progressively higher mortality: MPI $1=12.5 \%$ mortality, MPI $2=41.6 \%$ mortality, and MPI $3=83.3 \%$ mortality $(\mathrm{p}=0.001)$. Adjusting for age and sex, the prognostic efficacy of MPI for defining the risk of mortality was confirmed and was highly significant (odds ratio 10.47, 95\% CI 2.04-53.6).
Figure 1 shows the survival curves of patients with different grades of MPI: patients with a higher MPI demonstrated a significantly higher mortality rate $(\mathrm{p}=0.001)$.

\section{Discussion}

In this study we demonstrated that a CGA can be used in the clinical setting to stratify patients $\geq 65$ years hospitalized for an upper gastrointestinal bleeding according to their risk for 2-year mortality. Data obtained from a standardized CGA, including clinical, functional, cognitive, nutritional and social parameters, were used for calculating a MPI that was significantly associated with the risk of mortality in this population of older patients. Mortality was selected as an outcome because it is dichotomous, easily distinguishable, and unequivocal. The findings of this study demonstrate the importance of considering multidimensional impairment in prognostic systems for elderly hospitalized patients.

Inclusion of group of parameters relating to multidimensional aspects of elderly patients, obtained from a standardized CGA, yielded a multidimensional index, i.e., the MPI, which proved excellent in identifying three groups of 2-year mortality risk as demonstrated by the three distinct and uncrossed survival curves. This MPI was calculated from conventional CGA data easily col- 
lected from hospitalized elderly patients. Unlike the CGA used in community-dwelling or institutionalized elderly people $[15,16]$, in the present study we used parameters that particularly characterize hospitalized elderly patients, such as comorbidity and the number of medications taken.

As expected [17], a high number of patients with upper gastrointestinal bleeding were taking aspirin or NSAIDs $(30.5 \%)$ and/or warfarin (8.3\%). Moreover, of those patients who were taking these damaging drugs, only 2 were treated with proton pump inhibitors as preventative therapy, confirming the underutilization of pharmacological gastroprotection in older high-risk patients [18].

This multidimensional approach has been successfully used in hospitalized elderly patients for clinical [19] and, very recently, even for administrative purposes [20].
Indeed, a significant correlation was found between multidimensional impairments assessed by the CGA criteria and the all-patients-refined-(APR)-DRG system, which is an administrative tool useful in identifying elderly inpatients at high risk of high health-resource consumption.

In conclusion, CGA is a useful tool for evaluating older patients with upper gastrointestinal bleeding. For the first time, we describe a CGA-derived MPI that is a clinically useful tool for stratifying older patients into three different grades of 2-year mortality risk. These findings suggest that opportunity does exist to consider CGA a prognostic instrument clinically useful for the short- and long-term management of older patients with upper gastrointestinal bleeding.

\section{References}

1 Rubenstein LZ, Rubenstein LV: Comprehensive geriatric assessment of older patients with gastrointestinal disorders; in Pilotto A, Malfertheiner P, Holt PR (eds): Aging and the Gastrointestinal Tract. Interdiscip Top Gerontol. Basel, Karger, 2003, vol 32, pp 1227.

-2 Consensus Development Panel (Chairman: D Solomon): National Institutes of Health Consensus Development Conference Statement: Geriatric Assessment methods for clinical decision-making. J Am Geriatr Soc 2003;51:1490-1494.

3 Rubenstein LZ: Comprehensive geriatric assessment: from miracle to reality. J Gerontol Med Sci 2004;59A:473-477.

4 Cotton P, Williams CB: Practical Gastrointestinal Endoscopy, ed 3. Oxford, Blackwell Scientific, 1990.

$\checkmark 5$ Laine L, Peterson WL: Bleeding peptic ulcer. N Engl J Med 1994;331:717-727.

$\checkmark 6$ Katz S, Downs TD, Cash HR, Grotz RC: Progress in the development of an index of ADL. Gerontologist 1970;10:20-30.

$\checkmark 7$ Lawton MP, Brody EM: Assessment of older people: self-maintaining and instrumental activities of daily living. Gerontologist 1969; 9:179-186.
-8 Pfeiffer E: A short portable mental status questionnaire for the assessment of organic brain deficit in elderly patients. J Am Geriatr Soc 1975;23:433-441.

-9 Linn B, Linn M, Gurel L: The Cumulative Illness Rating Scale. J Am Geriatr Soc 1968;16: 622-626.

10 Guigoz Y, Vellas B: The Mini Nutritional Assessment (MNA) for grading the nutritional state of elderly patients: presentation of the MNA, history and validation. Nestlé Nutr Workshop Ser Clin Perform Programme 1999;1:3-11.

11 Bliss MR, McLaren R, Exton-Smith AN: Mattresses for preventing pressure sores in geriatric patients. Mon Bull Minist Health Public Health Lab Serv 1966;25:238-268.

12 L'informatore farmaceutico 2004: OEMF International, Milan 2002. Guidelines for ATC Classification. NLN Publ No 16. Uppsala, Nordic Council on Medicines, 1985.

13 Pilotto A, Franceschi M, Leandro G, D'Ambrosio PL, Scarcelli C, Corritore M, et al: Frailty Index from a Comprehensive Geriatric Assessment: a new tool to evaluate frailty in hospitalized elderly patients. J Nutr Health Aging 2006;10:327.

14 SPSS Version 13 Instruction Manual. Chicago, SPSS Inc, 2002.

$>15$ Carey EC, Walter LC, Lindquist K, Covinsky KE: Development and validation of a functional morbidity index to predict mortality in community-dwelling elders. J Gen Intern Med 2004;19:1027-1033.
16 Lee SJ, Lindquist K, Segal MR, Covinsky KE: Development and validation of a prognostic index for 4-year mortality in older adults. JAMA 2006;295:801-808.

17 Pilotto A, Franceschi M, Leandro G, Paris F, Niro V, Longo MG, et al: The risk of upper gastrointestinal bleeding in elderly users of aspirin and other non-steroidal anti-inflammatory drugs: the role of gastroprotective drugs. Aging Clin Exp Res 2003;15:494499.

18 Pilotto A, Franceschi M, Vitale DF, Zaninelli A, Masotti G, Rengo F on behalf of FIRI (Fondazione Italiana Ricerca suill'Invecchiamento) and the SOFIA Investigators: Upper gastrointestinal symptoms and therapies in elderly outpatients, users of non-selective NSAIDs or coxibs. Aliment Pharmacol Ther 2005;22:147-155.

19 Ellis G, Langhorne P: Comprehensive geriatric assessment for older hospital patients. $\mathrm{Br}$ Med Bull 2004;71:45-49.

20 Pilotto A, Scarcelli C, D’Ambrosio LP, Cascavilla L, Longo MG, Greco A: All patient refined diagnosis related groups: a new administrative tool for identifying elderly patients at risk of high resource consumption. J Am Geriatr Soc 2005;53:1-2. 